\title{
La motivación en el aprendizaje de la matemática: Perspectiva de estudiantes de
} básica superior

\section{Motivation in learning mathematics: Perspective of students of superior basic}

\author{
Lourdes Patricia Calle Chacón \\ lourdes.calle@psg.ucacue.edu.ec \\ Universidad Católica de Cuenca, Azogues \\ Ecuador \\ https://orcid.org/0000-0002-3597-7239 \\ Darwin Gabriel Garcia-Herrera \\ dggarciah@ucacue.edu.ec \\ Universidad Católica de Cuenca, Azogues \\ Ecuador \\ https://orcid.org/0000-0001-6813-8100 \\ Sergio Constantino Ochoa-Encalada \\ scochoae@ucacue.edu.ec \\ Universidad Católica de Cuenca, Cuenca \\ Ecuador \\ https://orcid.org/0000-0003-3067-3719 \\ Juan Carlos Erazo-Álvarez \\ jcerazo@ucacue.edu.ec \\ Universidad Católica de Cuenca, Cuenca \\ Ecuador \\ https://orcid.org/0000-0001-6480-2270
}

Recibido: 27 de abril de 2020

Revisado: 22 de mayo de 2020

Aprobado: 12 de junio de 2020

Publicado: 29 de junio de 2020 


\title{
RESUMEN
}

La investigación tiene como objetivo general analizar de qué manera influye la motivación de los estudiantes en el aprendizaje de la matemática de la Unidad Educativa Héroes de Verdeloma. De tipo descriptiva correlacional no experimental, calculándose el Chicuadrado de Pearson con la finalidad de conocer la asociación de las variables de estudios. Entre los resultados más relevantes se puede observar que a los estudiantes les gusta aprender temas de matemática y les agrada como el docente imparte sus clases, pero sin embargo el aprendizaje no llega de igual manera a los estudiantes, y no todos entienden los diferentes temas de matemática. No todos se sienten completamente motivados por aprender, además no llama su atención el investigar más sobre lo aprendido, es así que solo se quedan con lo aprendido del docente dentro del aula de clase, lo que puede limitar sus conocimientos.

Descriptores: Técnica didáctica; ambiente de la clase; actitud del estudiante; relación profesor-alumno. (Palabras tomadas del Tesauro de la UNESCO).

\begin{abstract}
The research has the general objective of analyzing how the motivation of students influences the learning of mathematics at the Héroes de Verdeloma Educational Unit. Non-experimental correlational descriptive type, calculating Pearson's Chi-square in order to know the association of the study variables. Among the most relevant results, it can be observed that students like to learn math topics and they like how the teacher teaches their classes, but nevertheless the learning does not reach the students in the same way, and not everyone understands the different topics of math. Not everyone is completely motivated to learn, and it is not their interest to investigate more about what has been learned, so they only keep what they have learned from the teacher in the classroom, which can limit their knowledge.
\end{abstract}

Descriptors: Classroom techniques; classroom environment; student attitudes; student teacher relationship. (Words taken from the UNESCO Thesaurus). 


\section{INTRODUCCIÓN}

Hoy en día, un problema muy común que se puede observar en el área de matemática, es que los estudiantes demuestran cierta resistencia a desarrollar procesos de enseñanza aprendizaje, pues le produce miedo a no entender y por ende no poder solucionar los ejercicios con los que se encuentren, o en algunos casos, simplemente no les gusta la asignatura. Por otro lado, los docentes necesitan estar en formación continua para mejorar la forma en la que activan los conocimientos en clase, a través de actividades motivadoras, que animen el interés y la motivación de los estudiantes para aprender matemáticas.

La motivación juega un rol muy importante en la escuela, por cuanto por medio de esta se puede determinar el rendimiento académico de las y los estudiantes, también, permite identificar los métodos que se ejecutan dentro del aula de clase para desarrollar competencias dentro del alumnado, (Alcade \& Hernández, 2017) por ello, el tener estudiantes motivados en las aulas de clase, puede generar mejores procesos de adquisición del contenido que se pretende impartir.

La motivación dentro del aprendizaje según (Ospina, 2006) contribuye el ambiente propicio para desenvolverse en el salón de clase, es un plus que motiva el aprendizaje, por lo tanto, la motivación pasa a ser primordial dentro del aula, en cuanto a la actividad académica se refiere. Es así, que la motivación permite que los estudiantes se mantengan despiertos e interesados en lo que están aprendiendo, pues se generan mejores relaciones intrapersonales.

De ahí la importancia de crear un ambiente de clases diferente, con enfoque interactivo, es decir que el docente y sus estudiantes tienen que hablar el mismo idioma en cuanto a la forma de llevar la clase, con actividades que despierten su interés y que quieran involuntariamente aprender mucho más de lo que reciben de su dicente, quien debe dejar a un lado el propósito de que sus estudiantes alcancen una buena calificación sino más bien debe preocuparse por cómo está trabajando para mantener a sus estudiantes motivados y que lo que aprendan realmente quede impregnando en su conocimiento. 
Lo ideal para un estudiante sería que su profesor, durante el proceso de aprendizaje tenga la capacidad de motivarlo, no es suficiente con presionar o en ocasiones con culpar de su falta de interés, y por ende del incumplimiento de sus actividades pedagógicas, por lo que no basta con aconsejar, con tratar de ser amables sino que lo esencial es buscar estrategias innovadoras, motivadoras, que vayan más allá de los conocimientos que el docente como profesional pueda tener, ponerse en la línea que a ellos les gustaría aprender, enmarcados a mejorar el aprendizaje. (Valenzuela, 2015)

Cabe agregar, la importancia de la motivación en todo aspecto, en este sentido (Batista, Gálvez, \& Hinojosa, 2010) destacan que dentro del proceso enseñanza aprendizaje la motivación permite encauzar el esfuerzo, el comportamiento del estudiante, claro que el objetivo sería que el estudiante tenga una mejor actitud y el docente alcance lo que tanto busca, resultados eficaces en el proceso de educación, en busca de perfeccionarla cada día más. A a lo largo de la historia han existido teorías que explican la importancia de la motivación:

1. Teoría de Hull que aporta a la teoría motivacional en relación a las necesidades humanas cuyo origen es biológico.

2. Teoría del Tolman, su teoría aporta a la relación entre el ser y el medio; además relaciona el conocimiento con las expectativas, esta contribución se mantiene en las ideas modernas acerca de la motivación en el proceso de enseñanza aprendizaje.

3. Teoría del doble factor de Herzberg a pesar de ser un aporte en el ámbito laboral, hace referencia que, si existe un trabajo motivador, dirigido por el impulso producirá relaciones internas en el individuo, por lo que el individuo tendrá la sensación de dominio del entorno de igual manera y no menos importante la ausencia de este factor produce insatisfacción.

4. Teoría de la Expectativa atribuida a (Valenzuela, 2015) elaborada para conocer los niveles de motivación de los individuos, específicamente em el ambito laboral destacada porque el esfuerzo de una persona que siempre está encaminado a alcanzar el éxito, a mayor expectativa habrá mayor motivación, frente a un trabajo 
se buscan una recompensa, aplicándolo en el ámbito educativo podemos tomarlo desde el punto en el que para motivar a nuestro estudiantes utilizaremos estrategias interactivas que como recompensa darán un buen resultado académico y al a vez la expectativa de aprender jugando.

Por otro lado, (Montoya, Dussán, Taborda, \& Nieto, 2018) señala que la motivación promueve el aprendizaje dentro de los estudiantes, permitiéndole alcanzar sus metas o logros. Para ello, es necesario fomentar estímulos para que los mismos se motiven en desarrollar nuevos procesos de aprendizaje. Además, (Estrada, 2016) menciona que la motivación forma parte en el desarrollo de competencias a través de diferentes etapas y utilizando distintos recursos.

Por ello la necesidad de considerar que estamos frente a estudiantes que crecen con la tecnología, por lo tanto, los recursos que se utilicen los docentes en clases deben ser elaborados con herramientas digitales que es lo que generalmente despierta y mantiene su interés activo. En este sentido, (Mora \& Ortiz, 2015) plantean que una estrategia para promover el aprendizaje de las matemáticas en los estudiantes, es la modelación. La misma que consiste en trabajar cooperativamente para resolver problemas de la vida cotidiana por medio de las matemáticas. Además, los docentes se enfrentan a retos en cuanto a la estrategia didáctica, pero al mismo tiempo lograron cambiar su perspectiva respecto al aprendizaje. Por tanto, el uso de estrategias didácticas es un reto para el docente, pero puede cambiar la perspectiva por aprender de los estudiantes.

Es por eso, que los procesos en la actualidad, deben adaptarse a las necesidades que cada estudiante plantea desde su realidad. Por lo que se debe considerar estrategias que impulsen la motivación por aprender y también, generar espacios para que el docente pueda planificar sus estrategias didácticas conectado con las distintas asignaturas.

Por otra parte, (Marcano, 2015) señala que, al aplicarse estrategias didácticas como el juego, se mejora promedio de aprobados de $78,86 \%$ en relación a aquellos estudiantes que no $(38,39 \%)$, ya que aumenta la motivación dentro del grupo de clases. Por lo cual el promedio de aprobados es mayor y se comprobó que aplicar una estrategia didáctica basada en el juego, estimula la motivación, mejora el aprendizaje y el rendimiento 
académico. Además (Galán, 2016) plantean que aplicar actividades lúdicas para la enseñanza de operaciones básicas, mejora los procesos matemáticos en los niños, también aumenta su motivación por seguir aprendiendo temas matemáticos y tienen la intención de vincular estos aprendizajes con otras asignaturas.

(Suárez, Fernández, Rubio, \& Zamora, 2016) mencionan que la regulación de los aprendizajes, en el área de la matemática está ligada a la motivación que los estudiantes presentan, indistintamente del gusto que tengan hacia la asignatura, puesto que, el tiempo de realización de actividades indica que tan motivados se encuentran los alumnos. Por lo tanto, es significativo conocer que cada estudiante tiene necesidades diferentes, hay algunos que aprenden a otro ritmo y forma, para poder estimular el aprendizaje se lo debe hacer mediante estrategias motivadoras.

Según (Galán, 2016) Las TIC, se considera un recurso indispensable para la motivación en los estudiantes, a pesar de que muchas veces únicamente se les asocia con la diversión y pasatiempo, cuya clave es el uso que se les da dentro del aula, que obviamente aportan una forma interesante de impartir conocimientos, utilizando herramientas de manejo común entre los jóvenes, como es el caso de Tablet, celulares que si le damos buen uso dentro del ámbito educativo podremos conseguir muy buenos resultados, es así como se da paso a un concepto de educación denominado Educación 3.0, que de hecho hoy en día se le relacionan con el uso de redes sociales, que incorpora a la tecnología de comunicación e información pero dentro de un ambiente académico. De acuerdo con (Pacheco-Carrascal, 2016) el docente es quien debe trabajar en el entorno que involucra el aprendizaje matemático-estudiante- motivación, debe buscar una estrategia que motiven el interés y por ende el aprendizaje por la matemática, tomando en cuenta que en los estudiantes existe en los chicos una alta de interés y su deseo por aprender. Teniendo en cuenta lo anterior, la importancia de analizar el cómo se está motivando al estudiante, las estrategias que se van a utilizar durante una clase, hacer que las clases sean divertidas, por cuanto por si solas no van a dar los resultados que esperamos por lo que siempre se debe contar con ese plus que cambie la dinámica 
en el aprendizaje, también es vital considerar el apoyo que reciben en el hogar y mucho más el que le brindan en la escuela.

Para alcanzar la calidad educativa es importante considerar Educación 3.0, que puede tener obstáculos como por ejemplo el lugar en dónde nos desarrollamos, la economía, entre otros aspectos, que actualmente se cree que, frente a cualquier situación, la necesidad de usar estos sistemas en la educación es cada día más común y sobre todo obligatorio. Según el aporte de (Montoya, Dussán, Taborda, \& Nieto, 2018) para lograr la innovación educativa es importante implementar la creación de videojuegos, mismos que son considerados como recursos lúdicos y divertidos, son una alternativa para la educación, los docentes que se encuentran aplicando este tipo de educación deben buscar también nuevos espacios llenos de creatividad y entusiasmo.

Aprovechando las facilidades que hoy nos brinda la tecnología, es muy fácil crear un juego, por ejemplo dentro de Sploder que no implica mayor complicación y que como beneficio, aporta a la construcción del conocimiento pero dejando el aburrimiento y la rutina a un lado y escalando los intereses que pueden tener los estudiantes por este tipo de juegos, y si navegamos por la red encontraremos un sin número de opciones de videojuegos para implementar en el aula de clases, promoviéndose un liderazgo para el auto aprendizaje (Urrego, 2020).

Es por ello que, las redes sociales con propósitos educativos se usan frecuentemente, para desarrollar el trabajo colaborativo y que mejor manera de hacerlo mediante medios utilizados por gran cantidad de estudiantes alrededor del mundo, no se estarían usando estas redes, únicamente con fines sociales sino también con la idea de construir conocimientos (Pérez, Ortiz, \& Briseño, 2015).

Según (Pérez, Ortiz, \& Briseño, 2015) la redes sociales como Facebook que nace en 2004, juegan un papel muy importante dentro de la educaión relacionada con redes sociales, pues no hace diferenciacióbnde conocimientos es un medio accecible y puede ser usado por cualquier persona sin importar su preparación o edad, por lo que dentro del un enfoque cognitivo permite explorar y desarrollar nuevas habilidades, si al llegar a un gran número de poblacion sin hacer inguna distinción bien podrían utilizarla los 
Revista Arbitrada Interdisciplinaria KOINONIA

Año 2020. Vol V. ํ⒈ Especial Educación

Hecho el depósito de Ley: FA2016000010

ISSN: 2542-3088

FUNDACIÓN KOINONIA (F.K). Santa Ana de Coro. Venezuela.

Lourdes Patricia Calle Chacón; Darwin Gabriel Garcia-Herrera; Sergio Constantino Ochoa-Encalada; Juan Carlos Erazo-Álvarez

docentes para compartir e indagar los conocimientos de sus estudiantes en un ambiente colaborativo.

Cabe también mencionar el uso de WhatsApp por un gran número de usuarios que actualmente en medio de esta emergencia sanitaria como lo es el COVID-19, lo disponen como medio de comunicacíon entre el docente y el estudiante, y en las áreas rurales es de hecho el único medio de comunicación para poder continuar con el proceso escolar, pues mediante esta red comparten tareas, lecciones y llevan a cabo actividades escolares con total normalidad.

Si mencionaramos las facilidades y beneficios que nos brinda la tecnología tenemos un sin número de opciones, dentro de las que encontramos didácticas interactivas, los vídeo juegos, que sin duda son de gran ayuda pues aporta diversión a más de aprendizaje, los juegos al azar muy faciles de utilizar en cualquier momento de la clase, ya sean a través de las redes o simplemente podemos hacerlo en forma física, brindado tanto al docente como al estudiante una forma de interacción muy variada (Saltos-Cedeño, VallejoValdivieso \& Moya-Martínez, 2020).

Si a las herramientas tecnológicas que podríamos utilizar, le agregamos la innovacion, es decir el cambio constante, para generar en los estudiantes el deseo permanente de aprender, no importa si es algo pequeño pero que sea diferente y de vida al ambiente educativo del cual somos parte diariamente, no caer en la monotonía es la clave, que de hacerlo los estaríamos conduciendo directamente al cansancio o aburrimiento.

En función de lo planteado, la investigación tiene como objetivo general analizar de qué manera influye la motivación de los estudiantes en el aprendizaje de la matemática de la Unidad Educativa "Héroes de Verdeloma".

\section{MÉTODO}

La investigación es de carácter descriptivo correlacional no experimental, calculándose el Chi-cuadrado de Pearson con la finalidad de conocer la asociación de las variables de estudios (Guerrero, Lenise Do Prado, \& Ojeda, 2016), por lo que se da la apertura a nuevas líneas de investigación con énfasis contextualizado a la realidad de cada 
participante dentro del proceso investigativo (Rojas, 2015), siendo de orden transversal, se realizó la recolección de datos en un solo momento (Müggenburg \& Pérez, 2007).

Cómo técnica se recurrió a la encuesta, misma que permite recolectar información general de la población de estudio, además describe de manera óptima percepciones macro de los sujetos de la investigación (Ther, 2004). Se aplicó una encuesta a un total de 216 estudiantes de octavo, noveno y décimo año de educación básica superior, para conocer las percepciones que tienen acerca de la enseñanza y el aprendizaje de la matemática.

Las respuestas de cada pregunta de la encuesta son cerradas y están basadas en la escala de Likert que según (Ospina, Sandoval, Aristizábal, \& Ramírez, 2005), esta es útil para identificar actitudes y es utilizada de manera usual, pues permite alcanzar niveles de confiabilidad y se es necesario pocos ítems. El Instrumento fue validado a través del coeficiente alfa de Cronbach con un valor de 0,972

El procesamiento de la información se realizó con la ayudad el software estadístico SPSS en su versión 20 (Ramón-Minchala, Erazo-Álvarez, Narváez-Zurita, \& Moreno, 2020). Finalmente se comprobó la relación entre variables con la prueba de chi-cuadrado, que desde la perspectiva de (Tinoco, 2008) es una prueba estándar para datos no paramétricos.

\section{RESULTADOS}

Al realizar la comparación entre las variables, el entendimiento de las matemáticas y el aprendizaje, lo cual nos permite identificar que al ser la significancia bilateral menor a 0.05, por lo que sí a los estudiantes les gusta aprender matemáticas es diferente con referencia a su nivel de entendimiento ver tabla 1. 
Revista Arbitrada Interdisciplinaria KOINONIA

Año 2020. Vol V. $\mathrm{N}^{\circ} 1$. Especial Educación

Hecho el depósito de Ley: FA2016000010

ISSN: 2542-3088

FUNDACIÓN KOINONIA (F.K). Santa Ana de Coro. Venezuela.

Lourdes Patricia Calle Chacón; Darwin Gabriel Garcia-Herrera; Sergio Constantino Ochoa-Encalada; Juan Carlos Erazo-Álvarez

\section{Tabla 1}

Relación entre aprendizaje matemático en relación con rendimiento académico

\begin{tabular}{lccc} 
& Valor & gl & Sig. asintótica (bilateral) \\
\hline Chi-cuadrado de Pearson & $488,215^{\mathrm{a}}$ & 16 &, 000 \\
Razón de verosimilitudes & 420,727 & 16 &, 000 \\
Asociación lineal por lineal & 194,142 & 1 &, 000 \\
N de casos válidos & 216 & &
\end{tabular}

Fuente: Elaboración Propia.

Así mismo se hizo una comparación entre la forma de enseñar del docente y la motivación dentro del aula de clases y de igual manera se da una significancia bilateral de 0.05 lo que concluye que la forma de enseñar del docente no es proporcional al nivel de motivación del estudiante.

\section{Tabla 2}

Relación entre forma de enseñar y motivación dentro del aula

\begin{tabular}{lccc} 
& Valor & gl & Sig. asintótica (bilateral) \\
\hline Chi-cuadrado de Pearson & $273,901 \mathrm{a}$ & 16 &, 000 \\
Razón de verosimilitudes & 295,183 & 16 &, 000 \\
Asociación lineal por lineal & 127,726 & 1 &, 000 \\
N de casos válidos & 216 & &
\end{tabular}

Fuente: Elaboración Propia.

En cuanto a los resultados en torno a si les gusta la forma en como su profesor dicta la clase de matemáticas dicta la clase, el $51,4 \%$ se encuentra totalmente de acuerdo, mientras que sólo un 4,2\% está totalmente en desacuerdo. Lo cual nos demuestra, que los estudiantes a pesar de gustarles la manera de impartir clases de su docente 
Revista Arbitrada Interdisciplinaria KOINONIA

Año 2020 . Vol V. $\mathrm{N}^{\circ} 1$. Especial Educación

Hecho el depósito de Ley: FA2016000010

ISSN: 2542-3088

FUNDACIÓN KOINONIA (F.K). Santa Ana de Coro. Venezuela.

Lourdes Patricia Calle Chacón; Darwin Gabriel Garcia-Herrera; Sergio Constantino Ochoa-Encalada; Juan Carlos Erazo-Álvarez

\section{Tabla 3}

Forma de enseñar del docente

\begin{tabular}{lcc} 
& Frecuencia & Porcentaje \\
De acuerdo & 72 & 33,3 \\
\hline En desacuerdo & 8 & 3,7 \\
Ni de acuerdo ni en desacuerdo & 16 & 7,4 \\
Totalmente de acuerdo & 111 & 51,4 \\
Totalmente en desacuerdo & 9 & 4,2 \\
Total & 216 & 100,0 \\
\hline
\end{tabular}

Fuente: Elaboración Propia

Con respecto a la información si los estudiantes entienden los temas que explica el docente de matemática la tabla 4 evidencia que el 37,5\% está de acuerdo, el 15,3\% señala que está totalmente en desacuerdo y el $12 \%$ está en desacuerdo lo que refiere a que el aprendizaje que explica el docente en esta materia no llega de igual manera a todos los estudiantes. A partir de esto, se puede observar que a pesar de que los estudiantes responden que les gusta como el docente imparte clases y les gusta los distintos temas, hay un porcentaje que señala no entender los temas dentro de la materia de matemática.

\section{Tabla 4}

Entendimiento de la matemática

\begin{tabular}{lcc} 
& Frecuencia & Porcentaje \\
\hline De acuerdo & 81 & 37,5 \\
En desacuerdo & 26 & 12,0 \\
Ni de acuerdo, ni en desacuerdo & 25 & 11,6 \\
Totalmente de acuerdo & 51 & 23,6 \\
Totalmente en desacuerdo & 33 & 15,3 \\
Total & 216 & 100,0 \\
\hline
\end{tabular}

Fuente: Elaboración Propia 
Con respecto a la pregunta de sí a los estudiantes les gusta investigar más sobre el tema que enseña el docente, la tabla 5 muestra, el 32,9\% está totalmente de acuerdo, mientras que el $22,2 \%$ de los estudiantes están totalmente en desacuerdo. Esto nos permite identificar que un porcentaje de los estudiantes, no buscan otras alternativas de aprendizaje sobre lo que les enseña el docente, solo se quedan con el conocimiento dentro del aula de clases.

\section{Tabla 5}

Investigar los temas de matemática

\begin{tabular}{lcc} 
& Frecuencia & Porcentaje \\
\hline De acuerdo & 64 & 29,6 \\
En desacuerdo & 17 & 7,9 \\
Ni de acuerdo, ni en desacuerdo & 16 & 7,4 \\
Totalmente de acuerdo & 71 & 32,9 \\
Totalmente en desacuerdo & 48 & 22,2 \\
Total & 216 & 100,0 \\
\hline
\end{tabular}

Fuente: Elaboración Propia

Al considerar que la mayor parte de estudiantes concibe que la motivación es importante dentro de los procesos de enseñanza y aprendizaje de la matemática, es necesario considerar lo planteado (Garrote, Garrote, \& Jiménez, 2016) quienes mencionan que para fomentar la motivación dentro del aula de clases, es necesario tener en cuenta el modelo constructivista en el cual el estudiante pasa a ser el actor principal de su aprendizaje y el docente es el encargo de fomentar la motivación a través de estrategias innovadoras.

Por otro lado, (Salgado, Leria, Pilar, Gajardo, \& Olivares, 2017) plantean que el docente debe inculcar en sus estudiantes la motivación para alcanzar logros planteados al inicio de la asignatura, además señalan que la mayoría de los estudiantes tienen como única meta el alcanzar notas altas. Es así, que se evidencia que una parte de los estudiantes están interesados por los temas, mientras que otros no, así mismo en la misma 
proporción se indica la motivación del grupo de estudiantes. Para ello es necesario indagar sobres los aspectos que les motivan a aprender y cuáles son los factores que crear una barrera para adquirir esa motivación por el aprendizaje (González, 2020). También, es importante mencionar que (Razeto, 2016) plantea que uno de los factores principales para generar motivación en los estudiantes, son los padres de familia, a partir de ellos se pueden identificar intereses que los estudiantes presentan y cómo ellos incentivan a los estudiantes para que se motiven. Es por ello, que, a través del interés por investigar temas relacionados con la asignatura de matemática, se debe considerar a los padres para que dentro de los hogares motiven a los alumnos a ir más allá de lo aprendido, navegar con profundidad en temas relacionados, así como también sea una guía para el docente y establezca puntos para generar esa motivación en el alumnado.

\section{PROPUESTA}

Por otro lado, es vital, tener en cuenta que aprender hoy en día debe involucrar un sin número de actividades motivadoras y creativas, abandonar la idea de que la motivación es solamente decir frases como "tu si puedes" "pon de tu parte", pues la motivación en la actualidad va mucho más allá, la motivación es innovar el aula de clase con la utilización de herramientas tecnológicas y juegos de azar que desarrollen competencias y que llamen la atención de los estudiantes.

Además, es necesario generar propuestas junto a los estudiantes sobre actividades que se puedan trabajar y creen en el estudiante un gusto por el aprendizaje, evitando así el aburrimiento o el no querer aprender alguna materia. 


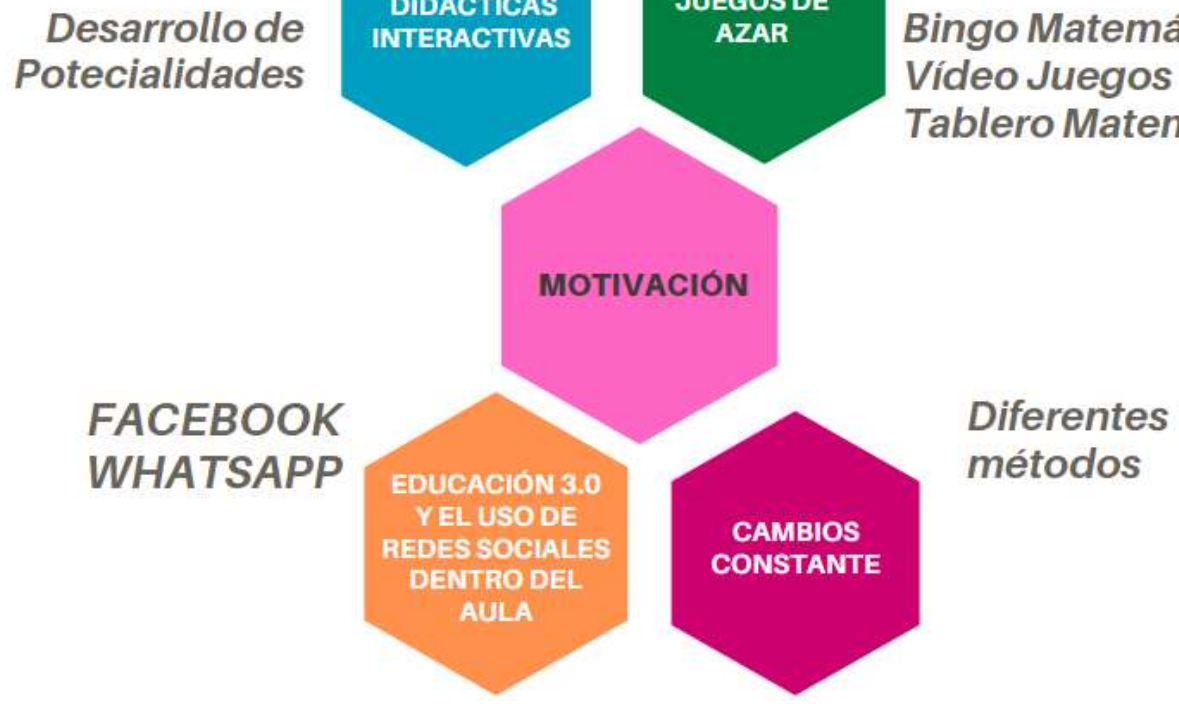

Figura 1. Motivación por el aprendizaje matemático.

Al identificar un problema sabemos que se nos abre una posibilidad para encontrar la solución, en este caso se identifica que el desinterés de los estudiantes por la matemática, no tiene que ver con que no les gusta la asignatura o la forma con la que su docente imparte la clase, sino es una situación más de fondo, pues al preguntarle a los estudiantes si se sentían motivados durante una clase de matemática, ellos no se sentían a gusto tenía expectativas por algo más, de ahí la propuesta de ir a la par de los estudiantes, tomando en cuenta que la mayoría de docentes son migrantes digitales, que conocen poco o nada de herramientas tecnológicas por lo que deberían estar a la vanguardia de la tecnología.

En la utilización de plataformas que permitan motivar al estudiante a aprender de la forma como ellos hoy en día lo hacen, sin olvidar que están frente a ciudadanos digitales, que poseen conocimientos innatos sobre tecnología, mal se haría con pretender motivar al estudiante con cosas o actividades que no llamen su atención, o con practicar el mismo 
método todos los días durante la clase, se puede incluir una forma de impartir clases mediante la utilización de lo que más disfrutan hacerlo.

Vídeo juegos como una herramienta para desarrollar sus potencialidades e impartir un conocimiento de forma más divertida, alejándonos de la idea que es una actividad dañina que generalmente es lo que se escucha de los padres de familia, está alternativa dentro de la educación, apuestan a retener el conocimiento con mayor facilidad, motivando al estudiante a verse inmerso en una competencia sana en dónde sentirá ganas de aprender más, se puede utilizar Sploder, Minecraft, Dragon Box, SimCity Edu, ClassDojo entre otros.

Juegos de azar que también contribuyen al trabajo colaborativo, logrando que se interesen en el aprendizaje porque ahora hacerlo les resultará placentero, podemos usar: Bingo matemático en la que el estudiante tiene la posibilidad de interactuar con sus compañeros pues al tener el ejercicio en la tabla de bingo deberá resolverlo para saber la respuesta y tomarlo como válido.

Tablero matemático muy parecido al conocido juego "monopolio", en donde se emplean dados, para poder iniciar la partida del juego, se puede hacerlo hasta con cuatro estudiantes quienes según el número que obtengan al lanzar el dado, irán resolviendo el ejercicio lo que les permitirá seguir avanzando, la condición sería que obtenga la respuesta y la posibilidad de acercarse a la meta será mayor.

Técnicas y didácticas interactivas que mientras se aplique se mantenga el interés, sin dar paso al aburrimiento, despertar en ellos la sensación de saber si lo hicieron bien, o si lo hicieron mal buscar nuevamente con mucha inquietud la forma de hacerlo bien esta vez. Existen varias opciones para crear este tipo de actividad, como Educaplay, Powtoon, Mindmeister, Powtoon entre otras.

Educación 3.0. y el uso de redes sociales se les considera como un nuevo modelo educativo, pues al ser la principal vía de comunicación es favorable utilizarla en formación académica, como una forma más dinámica de interactuar en el aula, da paso a debatir con otros usuarios sobre temas tratados en el aula, pero como todo, tiene la desventaja, 
pues, el estudiante que utilice libremente este sistema puede exceder el uso o en su defecto utilizarla como escudo para creer que se está aprendiendo algo.

Cambio constante o dicho de otra forma la innovación, todos hemos experimentado la sensación de probar algo nuevo e interesante, lo mismo sucede en la educación cuando traemos algo nuevo al aula de clases, los estudiantes se sentirán motivados y con más interés de lo habitual, por lo que está prohibido mantenerse con el mismo método o peor aún con las mismas clases aburridas y rutinarias.

\section{CONCLUSIONES}

Es importante, tener siempre presente que el motor que impulsa la conducta humana es la motivación, un estudiante motivado, dará lo mejor de sí, permitirá explotar sus potencialidades he ahí el trabajo del docente, padres de familia, impulsarlo positivamente para ello es necesario analizar y tomar en cuenta las diferentes perspectivas de los actores dentro de cualquier tipo de institución.

Desde los puntos de vista de los estudiantes se ha podido identificar este rol fundamental que cumple la motivación para ellos dentro de la enseñanza de la matemática. El docente y el estudiante están en constante aprendizaje, más en la sociedad que ese está revolucionando de manera constante.

Es evidente que la sociedad constantemente está avanzando, por tanto, los docentes, estudiantes, incluso padres de familia también deben hacerlo, meditando siempre que el aprendizaje es primordial dentro de los estudiantes y los mismos deben sentirse plenamente motivados para desarrollar sus actividades de manera oportuna.

Por lo tanto, es aconsejable trabajar mucho en la motivación pero acompañados de herramientas tecnológicas, para una buena clase no debe faltar actividades relacionadas con la web ya sea para implementarlas en el aula o para reforzar el aprendizaje en sus hogares, que se resistiría a aprender con un vídeo juego, si sabe que al equivocarse tiene otra oportunidad para divertirse jugando y a la vez aprendiendo, lo mismo sucede con el uso de las redes sociales, pero con fines educativos, en donde el docente es el encargado directo de utilizar estos medios para impartir conocimientos a los estudiantes 
desarrollando sus potencialidades, pues es una gran oportunidad para usar los canales de comunicación que la mayoría de ellos los utilizan, a pesar de que estos medios generalmente son considerados únicamente como ocio y diversión, son de gran beneficio en el campo educativo.

De esta manera se puede finalizar diciendo que un ambiente armónico y diferente, en constante cambio, siempre innovando, incluyendo en simples clases dinámicas, juegos de azar que mantengan al joven despierto y emocionado, evitando caer en la rutina será muy beneficioso no solo para el estudiante sino también para el docente, tener siempre presente que el arte de educar es una vocación que la podemos llevar a cabo con mucha pasión.

\section{FINANCIAMIENTO}

No monetario.

\section{AGRADECIMIENTO}

A la Universidad Católica de Cuenca, por impulsar y apoyar el desarrollo de la investigación.

\section{REFERENCIAS CONSULTADAS}

Alcade , R., \& Hernández, R. (2017). Estilos motivacionales y expectativas educativas en las familias inmigrantes: un análisis basado en el estudio de casos de familias inmigrantes de origen dominicano [Motivational styles and educational expectations in immigrant families: an analysis base]. Revista Complutense de Educación, 455-474. doi:10.5209/RCED.52795

Batista, S. A., Gálvez, E. M., \& Hinojosa, C. I. (2010). Bosquejo histórico sobre las principales teorías de la motivación y su influencia en el proceso de enseñanzaaprendizaje [Historical outline of the main theories of motivation and its influence on the teaching-learning process]. Revista General de Medicina, 26(2), 376-386. Obtenido de https://n9.cl/t8lr 
Estrada, A. (2016). Estrategias didácticas bajo el enfoque de competencias: aplicación del uso de herramientas de forma interactiva [Didactic strategies under the competence approach: application of the use of tools interactively]. RIDE Revista Iberoamericana para la Investigación y el Desarrollo Educativo, 398 - 411. Obtenido de https://n9.cl/ydar

Galán, J. G. (2016). Educación 3.0 en Iberoamérica: principales objetos de análisis científico y beneficios [Education 3.0 in Latin America: main objects of scientific analysis and benefits]. International Journal of Educational Research and Innovation, 124-145. Obtenido de https://n9.cl/1krv

Garrote, D., Garrote, C., \& Jiménez, S. (2016). Factores Influyentes en Motivación y Estrategias de Aprendizaje en los Alumnos de Grado [Influential Factors in Motivation and Learning Strategies in Undergraduate Students]. REICE. Revista Iberoamericana sobre Calidad, Eficacia y Cambio en Educación, 31-44. Obtenido de https://n9.cl/amp6

González, F. (2020). La gerencia del aula desde la vocación del colectivo docente. [Classroom management from the vocation of the teaching community]. EPISTEME KOINONIA, 3(5), 51-71. http://dx.doi.org/10.35381/e.k.v3i5.528

Guerrero, R., Lenise Do Prado, M., \& Ojeda, M. (2016). Reflexión crítica epistemológica sobre métodos mixtos en investigación de enfermería [Critical epistemological reflection on mixed methods in nursing research]. Enfermería Universitaria, 13(4), 246-252. Obtenido de https://n9.cl/hzj7r

Marcano, K. (2015). Aplicación de un juego didáctico como estrategia pedagógica para la enseñanza de la estequiometría [Application of a didactic game as a pedagogical strategy for teaching stoichiometry]. Revista de Investigación, 181-204. Obtenido de https://n9.cl/uffx

Montoya , D., Dussán, C., Taborda, J., \& Nieto, L. (2018). Motivación y estrategias de aprendizaje en estudiantes de la Universidad de Caldas [Motivation and learning strategies in students of the University of Caldas]. Tesis Psicológica, 1-23. Obtenido de https://n9.cl/x99no

Mora, A., \& Ortiz, J. (2015). Capacidades didácticas en el diseño de tareas con modelación matemática en la formación inicial de profesores [Teaching skills in the design of tasks with mathematical modeling in the initial training of teachers]. Perspectiva Educacional, Formación de profesores, 110-130. Obtenido de https://n9.cl/w21a 
Müggenburg, M., \& Pérez, I. (2007). Tipos de estudio en el enfoque de investigación cuantitativa [Types of study in the quantitative research approach]. Enfermería Universitaria, 35-38. Obtenido de https://n9.cl/r3ke1

Ospina, B., Sandoval, J., Aristizábal, C., \& Ramírez, M. (2005). La escala de Likert en la valoración de los conocimientos y las actitudes de los profesionales de enfermería en el cuidado de la salud. Antioquia, 2003 [The Likert scale in the assessment of knowledge and attitudes of nursing professionals in health care]. Investigación y Educación en Enfermería, 14-29. Obtenido de https://n9.cl/wne67

Ospina, J. (2006). La motivación motor del aprendizaje [The motor motivation of learning]. Revista Ciencias de la Salud, 4, 158-160. Obtenido de https://n9.cl/9r25

Pacheco-Carrascal, N. (2016). La motivación y las matemáticas [Motivation and mathematics]. Jour, 7, 149-158. Obtenido de https://n9.cl/fay7

Pérez, M. D., Ortiz, M. G., \& Briseño, M. M. (2015). Redes sociales en Educación y propuestas metodológicas para su estudio [Social networks in Education and methodological proposals for their study]. Ciencia, Docencia y Tecnología, 26(50), 188-206. Obtenido de https://n9.cl/gv9c

Ramón-Minchala, O. P., Erazo-Álvarez, J. C., Narváez-Zurita, C. I., \& Moreno, V. P. (2020). Integración de la responsabilidad social empresarial en proyectos de inversión dirigidos al sector agrícola [Integration of corporate social responsibility in investment projects aimed at the agricultural sector]. Revista Arbitrada Interdisciplinaria Koinonía, 702-728. doi:http://dx.doi.org/10.35381/r.k.v5i10.710

Razeto, A. (2016). Estrategias para promover la participación de los padres en la educación de sus hijos: el potencial de la visita domiciliaria [Strategies to promote parental involvement in their children's education: the potential of home visiting]. Estudios Pedagógicos, 449-462. Obtenido de https://n9.cl/9hkt

Rojas, M. (2015). Tipos de Investigación científica: Una simplificación de la complicada incoherente nomenclatura y clasificación [Types of Scientific Research: A Simplification of the Complicated Inconsistent Nomenclature and Classification]. REDVET. Revista Electrónica de Veterinaria, 1-14. Obtenido de https://n9.cl/sv6d

Salgado, J., Leria, F., Pilar, M., Gajardo, X., \& Olivares, M. (2017). Efecto de la Motivación sobre la Profundidad en los Procesos de Estudio en Universitarios de Formación en Pedagogía [Effect of the Motivation on the Depth in the Study Processes in University of Formation in Pedagogy]. REICE. Revista Iberoamericana sobre Calidad, Eficacia y Cambio en Educación, 87-105. Obtenido de https://n9.cl/gvrs 
Saltos-Cedeño, A., Vallejo-Valdivieso, P., \& Moya-Martínez, M. (2020). Innovación en educación matemática de básica superior durante el confinamiento por COVID19. [Innovation in higher basic mathematics education during confinement by COVID-19]. $\quad$ EPISTEME KOINONIA, $\quad 3(5), \quad$ 142-161. http://dx.doi.org/10.35381/e.k.v3i5.723

Suárez, J., Fernández, A., Rubio, V., \& Zamora, Á. (2016). Incidencia de las estrategias motivacionales de valor sobre las estrategias cognitivas y metacognitivas en estudiantes de secundaria [Incidence of motivational value strategies on cognitive and metacognitive strategies in high school students]. Revista Complutense de Educación, 421-435. Obtenido de https://n9.cl/hble

Ther, F. (2004). Ensayo sobre el uso de la encuesta: hermenéutica y reflexividad de la técnica investigativa [Essay on the use of the survey: hermeneutics and reflexivity of the investigative technique]. Revista Austral de Ciencias Sociales, 17-27. Obtenido de https://n9.cl/b7gor

Tinoco, O. (2008). Una aplicación de la prueba chi cuadrado con SPSS [An application of the chi square test with SPSS]. Industrial Data, 73-77. Obtenido de https://n9.cl/ha6b

Urrego, L. (2020). Liderazgo Estratégico: Perspectiva clave hacia la responsabilidad social en Instituciones de Educación Superior. [Strategic Leadership: Key perspective towards social responsibility in Higher Education Institutions]. Revista Arbitrada Interdisciplinaria Koinonía, 5(9), 28-43. http://dx.doi.org/10.35381/r.k.v5i9.556

Valenzuela, J. (2015). Motivación escolar: Claves para la formación motivacional [School motivation: Keys to motivational training]. Revista Electrónica de Motivación y Emoción, 356-367. Obtenido de https://n9.cl/7hkna

(C2020 por los autores. Este artículo es de acceso abierto y distribuido según los términos y condiciones de la licencia Creative Commons Atribución-NoComercial-Compartirlgual 4.0 Internacional (CC BY-NC-SA 4.0) (https://creativecommons.org/licenses/by-nc-sa/4.0/). 